\title{
Modélisation 3D d'un groupe de pieux pour le pont-canal de Houdeng
}

S. TREVISAN

X.L. LI

F. COLLIN

A. BOLLE

R. CHARLIER

Université de Liège Institut de Médecine

et de Génie civil

Chemin des Chevreuils

1. Bât. B52/3

B-4000 Liège 1

Belgique

C. COUNASSE

J.M. CREMER

J.Y. DEL FORNO

V. DE VILLE

Bureau d'étude Greisch
La prévision des tassements d'ouvrages hyperstatiques devient de plus en plus une donnée importante lors de leur dimensionnement et de leur réalisation.

Dans le cas du pont-canal de Houdeng (Belgique), nous avons abordé le problème de la connaissance du comportement des groupes de pieux et de la qualité de la réalisation des pieux forés.

Les auteurs décrivent ainsi une approche non classique du problème, par une analyse aux éléments finis basée sur des données expérimentales en nombre suffisant. Les objectifs principaux de cette étude sont la compréhension de l'influence, sur les tassements, d'un curage imparfait ou d'une destructuration de la roche en fond d'excavation lors de la réalisation des pieux forés ainsi qu'une meilleure perception des phénomènes impliqués dans le comportement d'un groupe de pieux.

Mots-clés : éléments finis, simulation 3D, tassements, pieux forés, groupes de pieux.

\section{Three dimensional modelization of the Houdeng canal-bridge piles group}

A satisfactory prediction of the settlements of hyperstatic structures is becoming more and more important in their design and construction. In this paper, the problems related to the behaviour of a group of piles and to the quality of the drilled piles of the canal-bridge of Houdeng (Belgium) are analysed in a non conventional fashion, based on a finite element analysis, thanks to sufficient experimental data.

The influence of an imperfectly cleaned excavation bottom and of the destructuration of the bottom rocks caused by the drilling of the plles is investigated. A particular attention is also given to the behaviour of a group of piles.

Key words : finite element, 3D simulation, settlement, drilled piles, group of piles. 


\section{Introduction}

Cette étude traite d'un domaine complexe et encore assez méconnu, dans lequel la littérature spécialisée présente une carence étonnante (Ottaviani, 1975 ; Desai et Muqtadir, 1986 ; Boulon, 1997). Il s'aqit d'un domaine dans lequel trouver des données expérimentales est difficile et pour lequel des investigations scientifiques n'ont pas encore été réalisées en suffisance : le comportement d'un groupe de pieux et l'influence du radier sur les déplacements et la distribution des contraintes dans le sol. Ce travail constitue une première étape de notre recherche; nous étudierons dès lors les phénomènes généraux qui caractérisent un groupe de pieux sous chargement, en analysant l'influence de chaque paramètre et la façon selon laquelle il contribue à la résistance finale et aux déplacements.

Pour étudier ces phénomènes, nous avons utilisé un code d'éléments finis permettant d'approcher au mieux le comportement des matériaux, grâce à des lois mécaniques sophistiquées. Le code de calcul LAGAMINE (Charlier, 1987) nous a donné la possibilité de faire des simulations bidimensionnelles mais également tridimensionnelles dans le domaine de la mécanique des sols, en abordant des problèmes complexes, dont la géométrie et les paramètres présentent une grande variabilité spatiale.

Nous avons étudié le problème particulier du pontcanal de Houdeng (Belgique), avec sa géométrie et ses caractêristiques en évaluant la possibilité d'adapter nos outils de calcul à des cas plus généraux. Ce sujet, particulièrement intéressant, nous a été proposé pour comprendre l'influence sur les tassements et sur la mobilisation des frottements latéraux d'une couche des débris sous la pointe des pieux. Ce matériau présentant de faibles caractéristiques mécaniques est le résultat du curage imparfait ou d'une destructuration en fond d'excavation, parfois observés lors de la réalisation des pieux forés.
Dans ce type de problème, la difficulté réside dans l'insuffisance de données relatives au comportement des ouvrages sous charge et l'impossibilité de faire des comparaisons entre les modèles numériques et la structure réelle. Toutefois, dans le cas du pont-canal de Houdeng, nous avons la possibilité d'obtenir ces données et de vérifier la fiabilité de notre outil.

\section{2}

\section{Le pont-canal de Houdeng}

Le pont (Fig. 1), réalisé en béton armé et précontraint, a une longueur totale de $498 \mathrm{~m}$, comptée entre les deux joints de dilatation disposés à l'avant des culées. Le tablier comporte 13 travées centrales continues de $36 \mathrm{~m}$ de portée, prolongées par deux travées d'extrémité en porte à faux de $15 \mathrm{~m}$. Les colonnes d'appui circulaires de $3 \mathrm{~m}$ de diamètre reposent sur des semelles carrées (épaisseur $3 \mathrm{~m}$ ) de $10 \mathrm{~m}$ de côté, fondées chacune sur 9 pieux forés tubés de $1,50 \mathrm{~m}$ de diamètre. Les charges reprises par les différentes colonnes sont de l'ordre de $60000 \mathrm{kN}$.

Notre travail et notre attention se concentreront en particulier sur l'étude du comportement des groupes de pieux sous les piles du pont. Ce thème est très actuel mais les recherches expérimentales disponibles aujourd'hui sont malheureusement insuffisantes pour approcher avec suffisamment de précision ce type de problèmes. Le calcul analytique de la résistance et des tassements d'un groupe de pieux se base sur des simplifications et des hypothèses fortes (Poulos, 1968; Viggiani, 1994: Frank, 1999); on perçoit donc l'importance que pourra avoir, dans le futur, la modélisation par éléments finis de ce type de fondation.

Les problèmes du pont-canal de Houdeng sont identiques à ceux de tous les ouvrages hyperstatiques et dérivent de la grande rigidité de ces structures. Les tassements différentiels sont mal tolérés et influencent fortement la redistribution des contraintes dans le

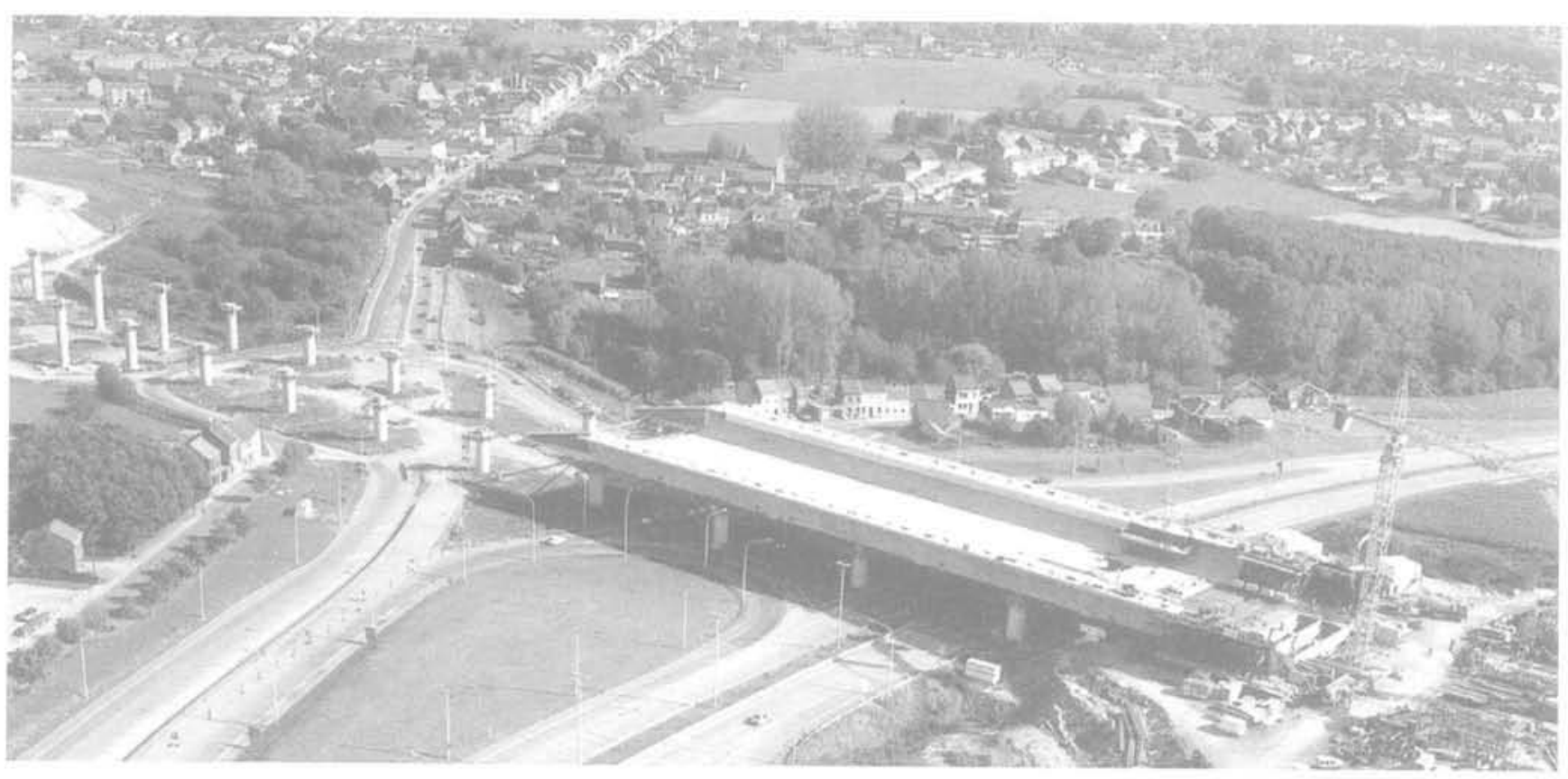

FIG. 1 Vue du chantier et vue en élévation du pont-canal de Houdeng. Sight of Houdeng canal-bridge yard. 
béton. En particulier dans le cas de l'ouvrage étudié, le tassement différentiel toléré est de $10 \mathrm{~mm}$ pendant toutes les phases de construction du pont et de seulement $5 \mathrm{~mm}$ à la fin du remplissage final du canal.

Tout cela se traduit dans la nécessité de mesurer à intervalles réguliers les tassements de chaque pile pendant toutes les phases de construction afin de pouvoir intervenir promptement et rester ainsi dans les limites imposées. Dans ce cadre, on peut comprendre l'importance de pouvoir disposer d'une prévision précise des tassements.

\section{3}

\section{Les données expérimentales disponibles}

Dans le cadre de la construction du pont-canal de Houdeng, un essai de chargement statique en vraie grandeur a été réalisé par le LCPC sur un pieu (h= $16,6 \mathrm{~m}-\mathrm{D}=0,90 \mathrm{~m}$ ) construit et instrumenté dans ce but. Le pieu résiste à la fois par frottement sur sa paroi latérale, en contact avec les terrains superficiels et la roche altérée, et par résistance à la pointe, encastrée dans le bedrock.

D'une part, l'évolution du frottement latéral dans les différentes couches du sol a été déduite des mesures enregistrées au cours de l'essai sur 10 tronçons référencés de bas en haut A, B, C, D, E, F, G, H et I, de longueurs respectives 1, 1, 1, 1, 2, 2, 2, 2 et $3,5 \mathrm{~m}$. Elle est donnée à la figure 2. D'autre part, l'interprétation des mesures fournit les deux courbes : charge en tête $Q_{0}-$ enfoncement de la tête $S_{0}$ et charge en tête $Q_{0}$ - enfoncement de la pointe $S_{p}$ (interprétation LCPC des mesures).

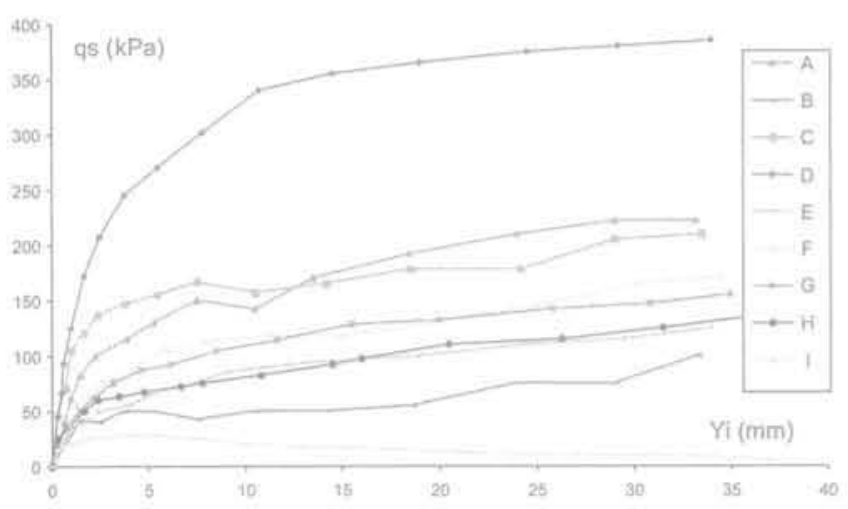

FiG.2 Évolution du frottement latéral q, en fonction du déplacement local Yi sur 10 troncons référencés de bas en haut A, B, C, D, E, F, G, H et I, de longueurs respectives $1,1,1,1,2,2,2,2$ et $3,5 \mathrm{~m}$ (mesures interprétées).

q. lateral friction evolution as a function of $\mathrm{Yi}$ local displacement on 10 soil layer sections Ireferences from bottom to top A, B, C. D. E. . . $\mathrm{G}, \mathrm{H}$ and 1 , length $1,1,1,1,2,2,2,2$ and $3.5 \mathrm{~m}$ (interpreted measures).

De plus, des essais pressiométriques ont été réalisés sur le site du pont-canal. Ceux-ci constituent la seule information relative au contexte géotechnique de l'ouvrage. On donne à la figure 3 les résultats provenant des deux sondages pressiométriques les plus proches du pieu d'essai.

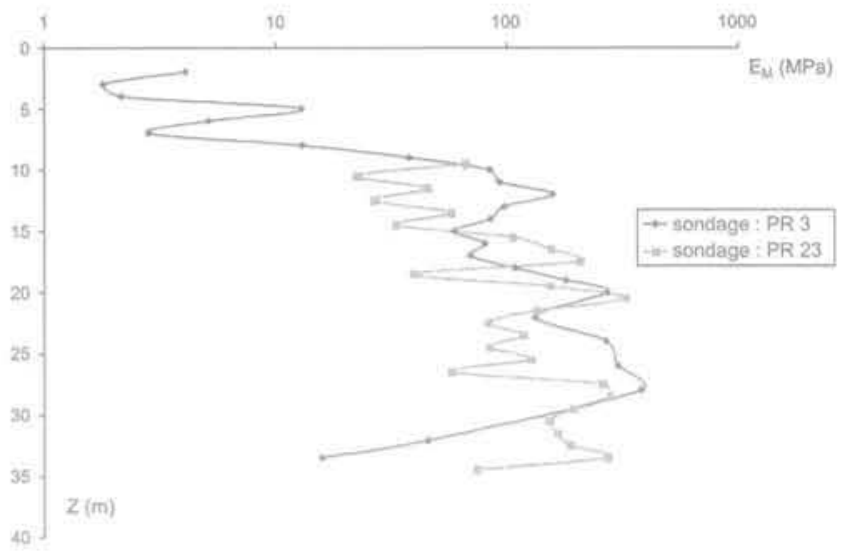

Fig. 3 Profils pressiométriques (module pressiométrique $\mathrm{E}_{\mathrm{M}}$ ).

Pressuremeter profiles ( $E_{u}$ pressuremeter modulus)

\section{Le profil géotechnique et les données utilisées}

Pour le calcul nous avons besoin de plusieurs données à introduire dans le code. Chaque matériau, chaque couche de sol (Fig. 4) doivent ètre caractérisés par leurs propriétés mécaniques. Les matériaux 2a et $2 \mathrm{~b}$ (situés sous la couche 1) désignent les schistes très altérés, tandis que les matériaux $3 \mathrm{a}$ à $3 \mathrm{~g}$, dans l'ordre de la profondeur, représentent les schistes plus ou moins altérés et fracturés.

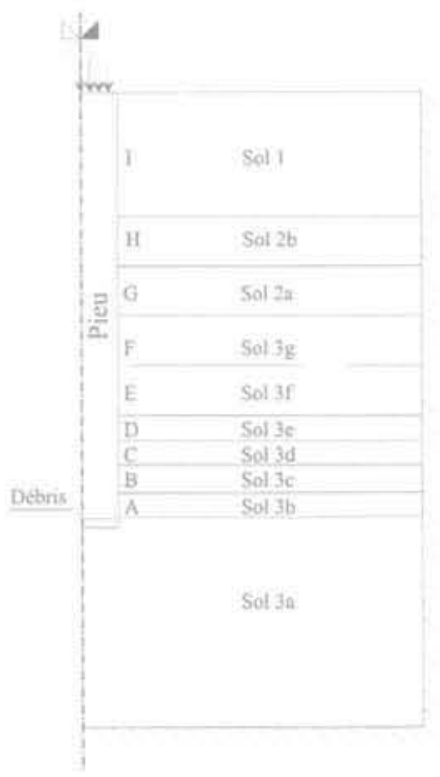

F1G.4 Idéalisation du problème, couches de sol. Problem idealization, soll layer.

Une partie de ces données est déduite des essais in situ, une autre partie sont des hypothèses de calcul. Par exemple le coefficient de Poisson, la dilatance, le choix de travailler avec un seul angle de frottement $\left(\phi=35^{\circ}\right)$ font partie des hypothèses de calcul. Le module de Young, déduit par la méthode pressiométrique, et la cohésion sont par contre des données découlant des essais in situ. 
Aux tableaux I, Il et III nous reportons les caractéristiques principales du béton, des débris, ainsi que des couches de sol discrétisées dans notre simulation. Les pieux et le sommier, en béton C $25 / 30$, ont été supposés élastiques isotropes. Les caractéristiques à court terme du béton constituant les pieux et le sommier sont données au tableau I. Pour les débris restant après curage ou destructuration de roche, nous considérons une couche de $20 \mathrm{~cm}$ d'épaisseur de matériau élastique très déformable (paramètres au tableau II).

Pour le sol nous avons utilisé une loi élastoplastique à frottement interne de Van Eekelen (Van Eekelen. 1980 ; Barnichon, 1998) (modèle élastique - parfaitement plastique),

L'état de contraintes initiales résulte du poids propre, avec un coefficient $K_{0}=1-\sin \varphi^{\prime}=0,43$ pour l'ensemble des matériaux, roches et béton (cette approximation simplifie fortement l'initialisation). La nappe aquifère est supposée au même niveau que le terrain. Le calcul est drainé.

TABLEAUI Paramètres du béton. Concrete parameters.

\begin{tabular}{l|c|c} 
& Symbole & Valeur \\
\hline Module de Young & $E_{b}$ & $26,1 \mathrm{Gpa}$ \\
\hline Coef. de Poisson & $v$ & 0,18 \\
\hline Masse volumique & $\rho_{b}$ & $2440 \mathrm{~kg} / \mathrm{m}^{3}$ \\
\hline
\end{tabular}

TABLEAU II Paramètres des débris. "Debris i parameters.

\begin{tabular}{l|c|c} 
& Symbole & Valeur \\
\hline Modile de Young & $\mathrm{E}_{\mathrm{b}}$ & $10 \mathrm{Mpa}$ \\
\hline Coef. de Poisson & $v$ & 0.35 \\
\hline Masse volumique & $\rho_{i}$ & $2440 \mathrm{~kg} / \mathrm{m}^{2}$ \\
\hline
\end{tabular}

\section{Le modèle numérique}

Le modèle numérique est la schématisation du système sol, pieu, interface sol-pieu, pourvu des diverses lois de comportement nécessaires pour représenter le problème dans sa globalité.

La résolution des équations d'équilibre implique une discrétisation du problème et sa division en un nombre fini d'éléments. Le milieu continu est discrétisé par des éléments solides et les interfaces entre deux différents matériaux sont discrétisées par des éléments de contact ou d'interface. Dans le code de calcul L.AGAMINE, les éléments finis solides 2D et 3D dont nous disposons sont des éléments isoparamétriques (Serendipity family, Zienkiewicz et Taylor, 1987). Pour les simulations 3D, nous avons utilisé des éléments à 8 nquuds, à champ de déplacement linéaire.

Pour modéliser les interfaces il est commode d'utiliser des éléments spécifiques, les éléments d'interface. accompagnés de lois constitutives adaptées. L'interface implique deux corps entre lesquels il y a une cinématique constituée essentiellement de glissements, mais aussi de gonflement ou d'amincissement dans le sens transversal. Les éléments d'interface se connectent aux éléments du solide. Ils doivent donc être compatibles avec la frontière des solides déformables (Habraken et Cescotto, 1986: Barnichon, 1998).

En considérant deux solides déformables $\Omega_{\text {, }}$, et $\Omega$, avec une surface extérieure $\partial \Omega_{U}$ et $\partial \Omega_{D}$, en contact entre

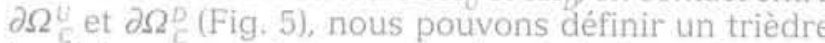
local $\left(e_{1}, e_{2}, e_{3}\right)$ à chaque point $S$ de la surface de contact. Dans ce système d'axe local, le tenseur des contraintes se réduit à un vecteur de contraintes de contact défini par trois composantes:

$$
\sigma_{c}=\left[\begin{array}{l}
\sigma_{1} \\
\sigma_{2} \\
\sigma_{3}
\end{array}\right]=\left[\begin{array}{l}
-\mathrm{p} \\
\tau_{2} \\
\tau_{3}
\end{array}\right]
$$

TABLEAUIII Les paramètres de chaque couche de sol. Soll layers parameters:

\begin{tabular}{|c|c|c|c|c|c|c|}
\hline Profondeur & $\begin{array}{c}\text { Couche êquivalente } \\
\text { de contact }\end{array}$ & $\begin{array}{l}\text { Nature } \\
\text { du sol }\end{array}$ & $E_{M}(\mathrm{kPa})$ & $\alpha$ & $\mathrm{E}(\mathrm{kPa})$ & $\mathrm{c}(\mathrm{kPa})$ \\
\hline$-16,6 m \rightarrow-26,6 m$ & & $3 a$ & \multirow{7}{*}{138000} & \multirow{7}{*}{$2 / 3$} & \multirow{7}{*}{207000} & 157 \\
\hline$-15,6 m \rightarrow-16,6 m$ & A & $3 b$ & & & & 157. \\
\hline$-14.6 \mathrm{~m} \rightarrow-15,6 \mathrm{~m}$ & B & $3 c$ & & & & 35. \\
\hline$-13,6 \mathrm{~m} \rightarrow-14,6 \mathrm{~m}$ & C & $3 \mathrm{~d}$ & & & & 150. \\
\hline$-12,6 \mathrm{~m} \rightarrow-13,6 \mathrm{~m}$ & D & $3 e$ & & & & 327. \\
\hline$-10,6 m \rightarrow-12,6 m$ & $E$ & $3 f$ & & & & 76. \\
\hline$-8,6 \mathrm{~m} \rightarrow-10,6 \mathrm{~m}$ & $\mathrm{~F}$ & $3 g$ & & & & 135. \\
\hline$-6.6 \mathrm{~m} \rightarrow-8,6 \mathrm{~m}$ & G & $2 a$ & \multirow{2}{*}{8500} & \multirow{2}{*}{$2 / 3$} & \multirow{2}{*}{13000} & 123. \\
\hline$-4,6 m \rightarrow-6,6 m$ & $\mathrm{H}$ & $2 \mathrm{~b}$ & & & & 117. \\
\hline $0 \rightarrow-4,6 \mathrm{~m}$ & 1 & 1 & 2700 & $1 / 2$ & 5400 & 15. \\
\hline Porosité $n=0,2$ & $\rho_{3}=2,8.10^{4} \mathrm{~N} / \mathrm{m}^{3}$ & $v=0,35$ & $\phi_{\mathrm{i}}=35^{\circ}$ & $\psi=0^{\circ}$ & & \\
\hline
\end{tabular}


où p est la pression, $\tau_{2}$ et $\tau_{3}$ sont les contraintes tangentielles dans les directions 2 et 3 . Ce vecteur des contraintes est défini dans un système local attaché à l'élément solide et il est indépendant de la rotation rigide du corps. La condition de contact parfaitement collant est traduite au niveau numérique en utilisant la méthode classique de pénalisation, qui autorise une petite vitesse relative entre les points $S^{U}$ et $S^{D}$ (une petite pénétration entre les deux solides) et un glissement relatif entre ceux-ci.

Le contact entre chaque corps $\Omega_{U}$ et $\Omega_{D}$ est discrétisé avec des éléments d'interface isoparamétriques, compatibles avec les éléments solides utilisés pour discrétiser les corps correspondants (Fig. 6). Les éléments de contact utilisés sont basés sur des principes variationels mixtes (Charlier et Habraken, 1990 ; Cescotto et Charlier, 1993) : les contraintes de contact sont calculées aux points d'intégration des éléments d'interface, alors que les déplacements du corps rigide sont calculés aux noeuds. Cette formulation entraîne une condition de contact plus douce que celle basée sur les conditions de contact aux nouds. La condition de contact est obtenue simplement au niveau loca' $2 \Omega^{\prime}$ le calcul de la distance $\lambda$ entre les deux interfaces ${ }^{\partial} \Omega_{c}$ et $\partial \Omega^{D}$

$$
\begin{gathered}
\lambda_{\mathrm{c}}<0 \Rightarrow \text { absence de contact } \\
\lambda_{\mathrm{c}} \geq 0 \Rightarrow \text { contact }
\end{gathered}
$$

Dans le code de calcul LAGAMINE, la surface de référence $\partial \Omega_{r}$ sur laquelle sont calculées les contraintes tangentielles fait toujours référence au côté oủ sont définis les éléments d'interface. L'autre surface de contact est discrétisée en utilisant des éléments qui per- mettent de simuler le contact entre deux solides déformables.

Pour le comportement frottant, nous avons adopté une loi de Coulomb:

$$
f=\sqrt{\left(\tau_{2}\right)^{2}+\left(\tau_{3}\right)^{2}}-(c+\mu p)
$$

avec $\mu=\tan \phi$ (coefficient de frottement) et $p$ la pression de contact. Dans cette relation $f$ est considéré comme une surface de plasticité dans le plan $\left(p \cdot \sqrt{\left(\tau_{2}\right)^{2}+\left(\tau_{3}\right)^{2}}\right)$, où nous pouvons définir trois zones (Fig. 7) :

$f<0$ : domaine de contact collant, absence de glissements ;

$f=0$ : domaine de contact glissant ;

$f>0$ : impossible.

Si $f<0$, pour un contact idéal, la variation du taux de déformation doit être nulle $\left(\dot{\varepsilon}_{-}=0\right)$. De toute façon, comme on l'explique plus loin, cette condition est adoucie en utilisant la méthode de pénalisation et en s'appuyant sur les observations expérimentales.

Le taux des contraintes de contact est calculé avec l'expression suivante:

$$
\dot{\sigma}_{\bar{C}}=\mathrm{K} \dot{\varepsilon}_{C}
$$

ou

$$
\left\{\begin{array}{l}
\dot{\mathrm{p}} \\
\dot{\tau}_{2} \\
\dot{\tau}_{3}
\end{array}\right\}=\left[\begin{array}{ccc}
K_{p} & 0 & 0 \\
0 & K_{\tau} & 0 \\
0 & 0 & K_{\tau}
\end{array}\right]\left\{\begin{array}{l}
\dot{\varepsilon}_{C 1} \\
\dot{\varepsilon}_{C 2} \\
\dot{\varepsilon}_{C 3}
\end{array}\right\}
$$

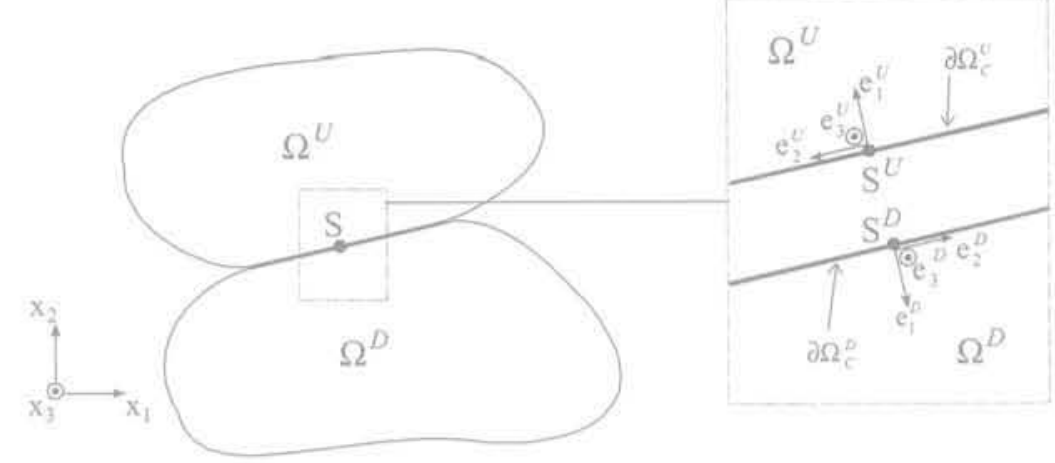

FGG.5 Contact entre solides déformables. Contact between deformable solids.

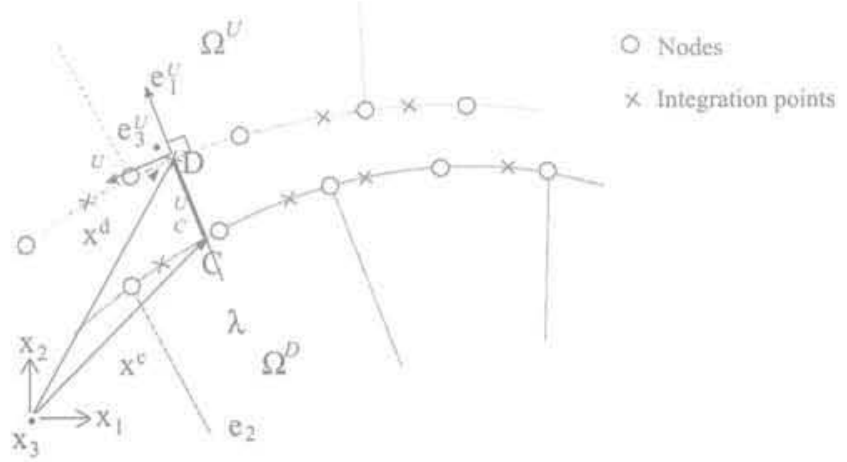

FG.6 Élément de contact parabolique (2D). Parabolic contact elements (2D).

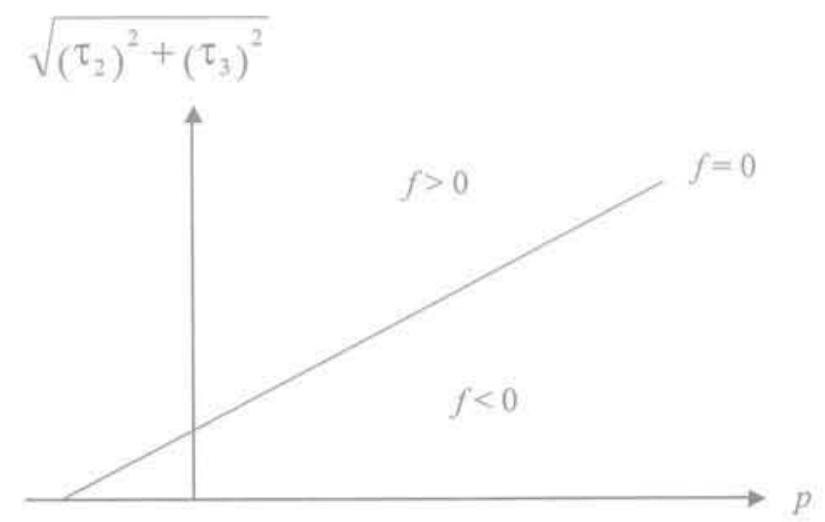

FiG.7 Loi de contact de Coulomb. Coulomb contact law. 
$K_{C}$ étant la matrice de pénalisation. Les coefficients de pénalisation $K_{p}$ et $K_{\mathrm{r}}$ contrôlent la pénétration tolérée $\varepsilon_{\text {a }}$ et le glissement relatif d par les formules approximatives:

$$
K_{p} \approx \frac{p}{\varepsilon_{c-1}}, K_{T} \approx \frac{\tau_{2}}{d}=\frac{\tau_{3}}{d}
$$

D'un point de vue pratique, les coefficients de pénalisation doivent être aussi grands que possible, pour prévenir une interpénétration significative et des glissements relatifs entre les deux corps. Cependant, une valeur trop importante peut conduire à des difficultés numériques de convergence. Par conséquent bien que la précédente équation donne des bons résultats, une certaine pratique est nécessaire pour choisir des valeurs acceptables pour $K_{p}$ et $K_{i}$

Dans le cas étudié nous avons relevé que le paramètre le plus influent dans la mobilisation du frottement est $K_{\tau}$ (raideur tangentielle). Il caractérise le début des courbes de mobilisation du frottement (Fig. 2), et n'est, expérimentalement, pas nul (au contraire du cas du contact idéal). Il est supposé ici proportionnel à la cohésion. On a ainsi :

$$
K_{\mathrm{r}}=\beta \mathrm{c}
$$

où $\beta$ est un coefficient à déterminer par la méthode itérative d'essais et d'erreurs, dans le but de reproduire lé mieux possible la mobilisation du frottement latéral. Nous avons considéré plusieurs jeux de paramètres et nous reportons (Tableau IV) les paramètres que nous avons choisis, lesquels ont ensuite été utilisés pour la simulation du groupe de pieux.
TABLEAU IV Jeu de $\mathrm{K}$, utilisé dans la simulation du groupe de pieux.

$\mathrm{K}_{\mathrm{T}}$ for the simulation of pile group.

\begin{tabular}{c|c|c}
\hline Couche & $c(\mathrm{kPa})$ & $\begin{array}{c}\mathrm{K}_{\mathrm{T}} \\
(\mathrm{Pa} / \mathrm{m})\end{array}$ \\
\cline { 2 - 3 } A & 157. & $1,20 \cdot 10^{9}$ \\
\hline B & 35. & $2,68.10^{7}$ \\
\hline C & 150. & $1,15.10^{8}$ \\
\hline D & 327. & $2,50.10^{4}$ \\
\hline E & 76. & $5,81.10^{7}$ \\
\hline F & 135. & $1,03.10^{8}$ \\
\hline G & 123. & $9,40.10^{7}$ \\
\hline H & 117. & $8,94.10^{5}$ \\
\hline I & 15. & $1,15.10^{7}$ \\
\hline
\end{tabular}

Le coefficient de pénalisation normale a été choisi égal à $K_{p}=11 \mathrm{MPa} / \mathrm{m}$ pour toutes les couches.

\section{6}

\section{Les cas étudiés et la procédure de travail}

La méthode que nous avons suivie dans notre travail a comporté une succession de simulations, à partir des cas les plus simples pour arriver à la simulation 3D du groupe de pieux. Notre parcours a commencé par l'étude du pieu modèle, d'un diamètre de $90 \mathrm{~cm}$, réalisé in situ et pour lequel nous disposons des résultats de l'essai de chargement statique (Fig, 2).

Nous avons réalisé des simulations numériques de ce pieu pour tarer le système, c'est-à-dire pour déterminer un jeu optimal de paramètres de comportement mécanique, pour chercher la meilleure combinaison de données à introduire dans le code. L'angle de frottement interne, la cilatance, le coefficient de Poisson, le coefficient de pénalisation normale, l'état de contraintes initial... sont des hypotheses de calculs. Les modules de Young et les cohésions ont été déduits directement des résultats expérimentaux. Le module initial de frottement à l'interface $K_{t}(6)$ a été calibré de façon à simuler au mieux l'essai de pieu.

Nous sommes partis avec de simples simulations 2D axisymétriques pour arriver finalement aux simulations 3D qui seront illustrées dans les paragraphes suivants. Nous avons également réalisé des simulations bidimensionnelles (Li et al, 2000) pour le groupe de pieux, en faisant des simplifications sur la géométrie du modèle. Cette étude nous a donné la possibilité de comprendre les limites d'un tel travail et l'importance d'une étude 3D en mécanique des sols, la seule qui nous donne la possibilité d'analyser des situations compliquées pour la géométrie et la disposition réelle des charges. Seul un calcul 3D nous permet de comprendre au mieux les mécanismes réels qui se développent dans des systèmes complexes comme ceux des groupes de pieux.

\section{1}

\section{Le pieu modèle, maillage 3D}

Le maillage du terrain est basé d'une part sur la description lithologique et, d'autre part, sur les résultats de l'essai de chargement statique en vraie grandeur sur le pieu modèle. Cela permet ainsi d'obtenir une bonne modélisation du frottement mobilisé par une prise en compte des caractéristiques mécaniques (essentiellement la cohésion) des différentes couches de roche et de sol. Le maillage s'étend à $10 \mathrm{~m}$ de profondeur sous la pointe du pieu, et à $10 \mathrm{~m}$ en largeur. La longueur modélisée du pieu est de $16,6 \mathrm{~m}$. Le niveau de la nappe aquifère correspond au niveau de la surface du terrain naturel.

Nous avons décidé de créer le maillage par rotation d'un modèle 2D autour de son axe de symétrie ; nous obtenons ainsi un octogone à la place d'une circonférence (Fig. 8: un quart du pieu). Ce choix est la conséquence d'une étude de l'influence de différents maillages, plus ou moins raffinés, sur les résultats au point de vue de la mobilisation du frottement et des tassements. Ceci nous a conduits à réaliser un maillage, composé de 348 éléments solides à 8 nœuds, 68 éléments d'interface verticaux, et 2 éléments de chargement mécanique statique. Le choix de travailler avec ce maillage est lié aux temps de résolution, particulièrement longs pour les simulations 3D. 


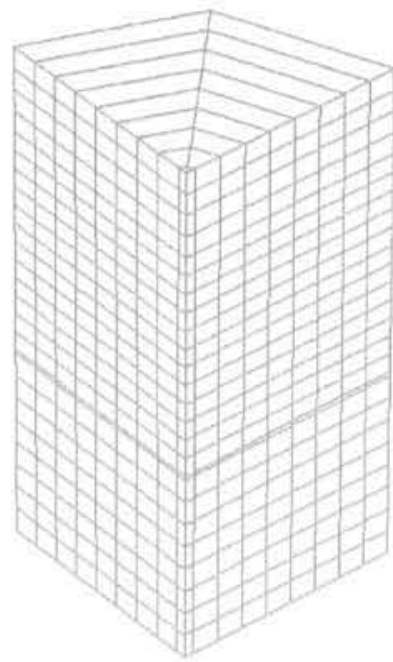

Fig. 8 Maillage du pieu modèle. Model pile mesh.

La charge statique est transmise à la tête du pieu par des éléments de chargement qui appliquent une charge de $400 \mathrm{kN}$ au premier pas. Le chargement augmente en 20 pas pour arriver à la charge maximale de $8000 \mathrm{kN}$.

Pour chaque pas de chargement, nous avons contrôlé les tassements à la tête et à la pointe du pieu, la mobilisation du frottement en fonction du déplacement relatif pieu-sol, et l'évolution de la contrainte à la base du pieu.

A la figure 9 nous avons reporté l'évolution des tassements mesurés et calculés à la tête et à la pointe du pieu en fonction du chargement. Comme nous avons déjà pu le constater dans le pieu d'essai, la raideur diffère entre les faibles et les fortes charges, Le comportement du pieu modélisé dans la simulation 3D sousestime la raideur en début et en fin de mise en charqe et met en évidence une transition entre la pente initiale et la pente finale qui apparaît trop tardivement dans les simulations. Lé tassement final à la pointe et à la base est parfaitement reproduit.

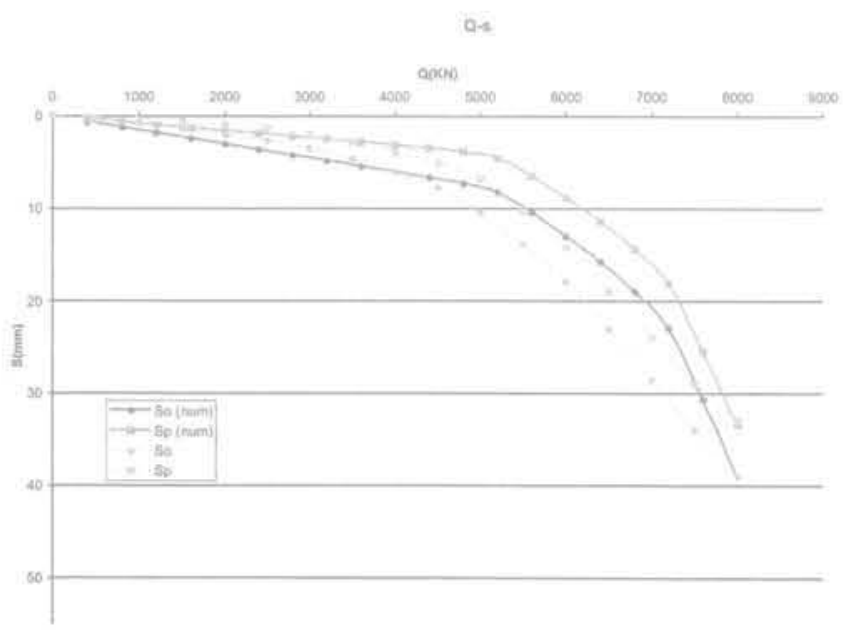

FiG.9 Charge en tête $\mathrm{Q}_{0}$ : tassement en tête $\mathrm{S}_{0}$ ou en base $S_{\text {. }}$.

Load at the head of pile $Q_{0}$ - settlement at the head $\mathrm{S}_{0}$ or at the base $\mathrm{S}_{\mathrm{p}}$.
Les figures 10, 11 et 12 montrent la mobilisation du frottement latéral vertical mesuré et calculé en fonction du déplacement relatif, pour chaque couche de sol. Nous pouvons remarquer la difficulté de s'approcher des courbes extrapolées à partir des résultats d'essai.
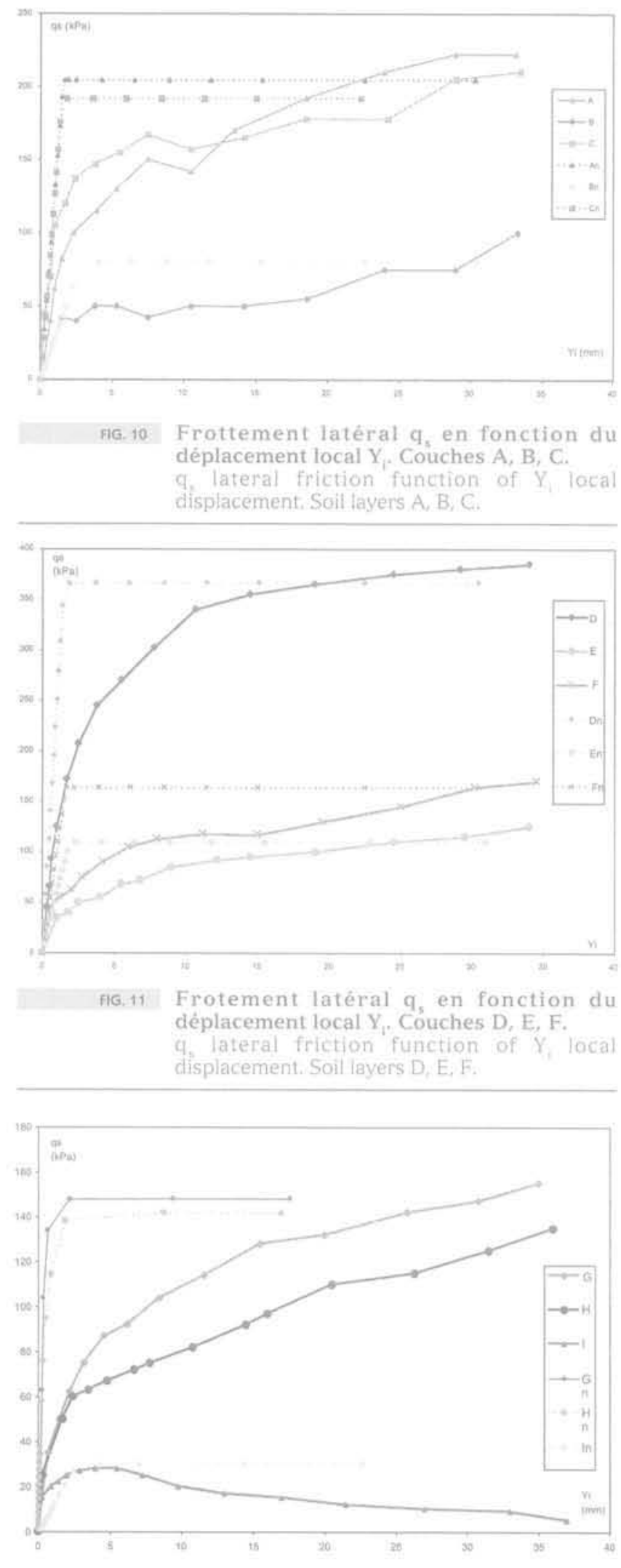

FG. 12 Frottement latéral $q$, en fonction du déplacement local X. Couches G, H, I.

$q$, lateral friction function of $Y_{\text {f local }}$ displacement - soil layers G, H, I. 
Nous observons des différences pour chaque couche entre la courbe d'essai et la courbe numérique. Toutefois, il reste utile d'étudier le comportement du groupe de pieu avec ces paramètres car ils représentent le comportement de manière tout à fait satisfaisante. Dans ce contexte, nous avons l'intention d'introduire ultérieurement une loi de comportement qui tient compte de l'écrouissage, afin de simuler au mieux le comportement de l'interface pieu-sol.

Les deux graphiques suivants montrent l'évolution de la charge en base $Q_{p}$ en fonction de la charge en tête $Q$ (Fig. 13) et des tassements en base $S$ en fonction de la contrainte en base $\sigma$. (Fig. 14). La transmission de la charge en base $Q_{\text {p }}$ en fonction de la charge en tête est surévaluée au début de la mise en charge et sous-êvaluée en fin de chargement. Nous remarquons le bon comportement du modèle 3D avec une loi de type Coulomb.

$\ln (\sin )$

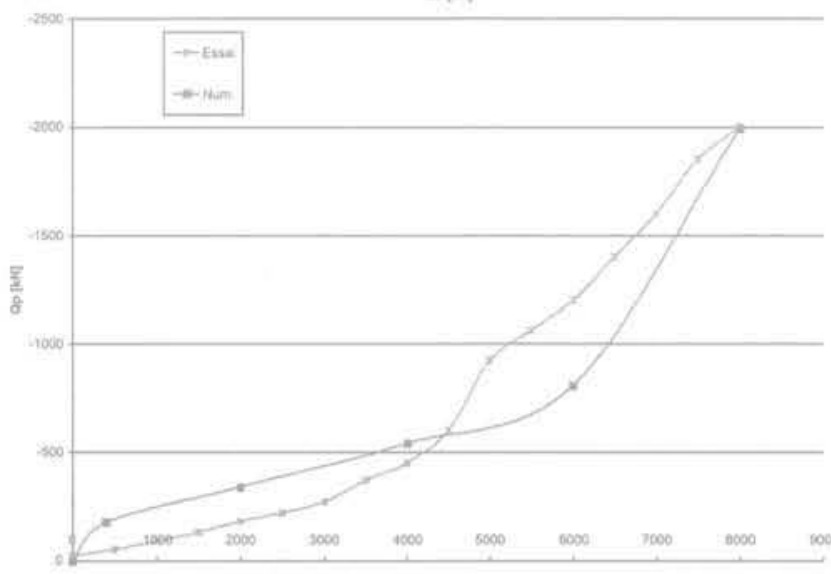

FIG. 13 Evolution de la charge en base $Q_{p}$ en fonction de la charge en tête $Q_{0}$.

Charge evolution at the base $\left(Q_{2}\right)$ function of the head load $(Q)$ :

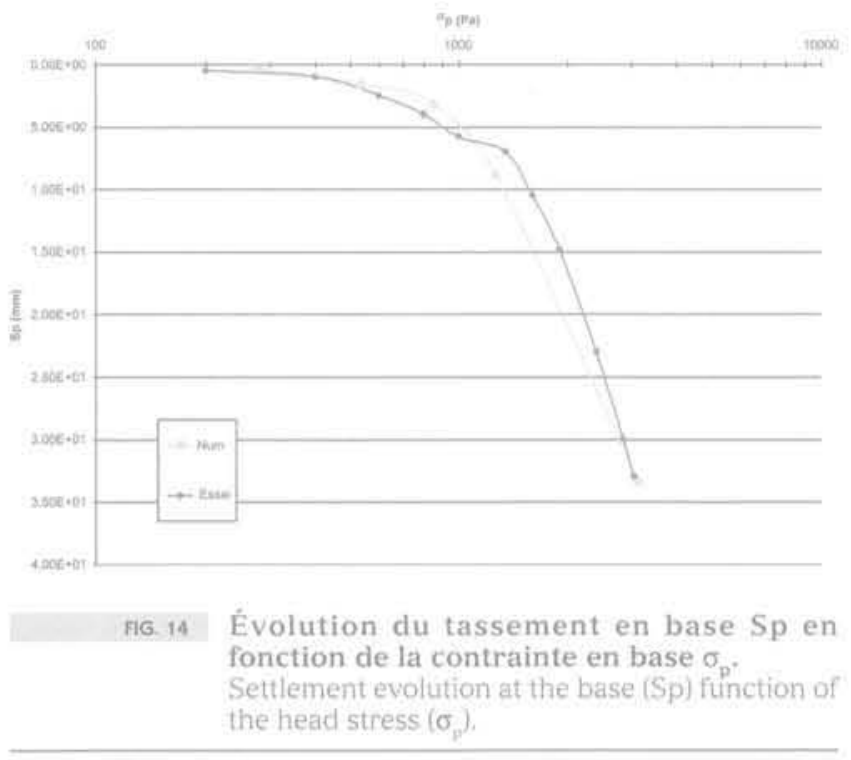

\section{Le groupe de pieux}

Le groupe de pieux du pont-canal a été étudié au début par un modèle 2D en simulant les pieux latéraux par une couronne circulaire (Li et al., 2000). Cette sim- plification, et les doutes qui en découlent, nous ont poussé à créer un maillage $3 \mathrm{D}$ avec la géométrie réelle du système. Cette modélisation 3D présente bien des avantages car elle offre la possibilité d'étudier des situations de chargement les plus variées et asymétriques. Dans notre simulation nous n'avons pas considéré l'ensemble du groupe de pieux, mais seulement un quart afin d'avoir un temps de calcul acceptable.

La réalisation du maillage a constitué le problème le plus difficile à résoudre. Il est composé de 1919 éléments solides, 306 éléments d'interface et 5 éléments de surface de chargement statique. Il est obtenu à partir d'un maillage plan par translation verticale en 19 couches d'éléments sur la hauteur (Fig. 15). Pour ce calcul nous avons utilisé les lois et propriétés déterminées dans la phase de tarage du pieu modẻle. Un sommier de $3 \mathrm{~m}$ d'épaisseur relie les pieux. Ceux-ci ont une longueur de $13,6 \mathrm{~m}$, leur pointe se trouve donc à la même profondeur que celle du pieu modèle.

Toutefois, en simulant uniquement un quart de la pile, nous avons quand même la possibilité de considérer différentes distributions du débris sous les pointes des pieux. Ce matériau, avec de faibles caractéristiques mécaniques (résidu d’un curage imparfait du fond d'excavation), peut se trouver sous les pointes des quatre pieux du modèle. En particulier, en faisant référence a la figure 16, dans la suite du travail nous appellerons a débris 1 n le débris situé sous la pointe du pieu central (le quart de pieu en bas), " débris 2 n celui sous le demi-pieu périphérique à droite, " débris 3 » celui sous le demi-pieu périphérique à gauche, (c débris 4 ) celui sous les pieux périphériques entiers.

Nous avons réalisé plusieurs séries de simulations en considérant différents emplacements des débris sous la pointe des pieux; en particulier, nous avons étudié les cas qui sont reportés dans le tableau V. La charge maximale de $100000 \mathrm{kN}$ est atteinte en 10 pas de $10000 \mathrm{kN}$. On remarque que la charge de service est de $60000 \mathrm{kN}\left(6^{\circ}\right.$ pas). Dans la suite, nous reporterons les résultats des simulations, en cherchant à faire des comparaisons pour comprendre comment la présence des débris influence les tassements de la pile et comment le radier contribue á la redistribution des charges dans le sol. De plus, nous discuterons une partie des

TABLEAUV Présence des débris sous la pointe de chaque pieu. "Debris" disposition below the pile head.

\begin{tabular}{c|c|c|c|c}
\hline $\begin{array}{c}\text { Presence } \\
\text { des débris }\end{array}$ & Pieu 1 & Pieu 2 & Pieu 3 & Pieu 4 \\
\hline Simulation 1 & OUI & OUI & OUI & OUI \\
\hline Simulation 2 & NON & NON & NON & NON \\
\hline Simulation 3 & OUI & NON & NON & NON \\
\hline Simulation 4 & NON & OUI & OU & OUI \\
\hline Simulation 5 & OUI & NON & NON & OUI \\
\hline Simulation 6 & NON & OUI & OUI & NON \\
\hline Simulation 7 & NON & NON & NON & OUI \\
\hline Simulation 8 & OUI & OUI & OU1 & NON \\
\hline Simulation 9 & OU1 & NON & OU1 & NON \\
\hline Simulation 10 & NON & OUI & NON & OUI \\
\hline
\end{tabular}




\begin{tabular}{|c|c|c|}
\hline $\begin{array}{c}\text { Éléments solides } \\
\text { Béton }\end{array}$ & $\begin{array}{c}\text { Éléments de contact } \\
\text { Sol-Béton }\end{array}$ & $\begin{array}{c}\text { Éléments solides } \\
\text { Sol }\end{array}$ \\
\hline S & & \\
\hline
\end{tabular}

FG. 15 Éléments du maillage. Mesh elements.

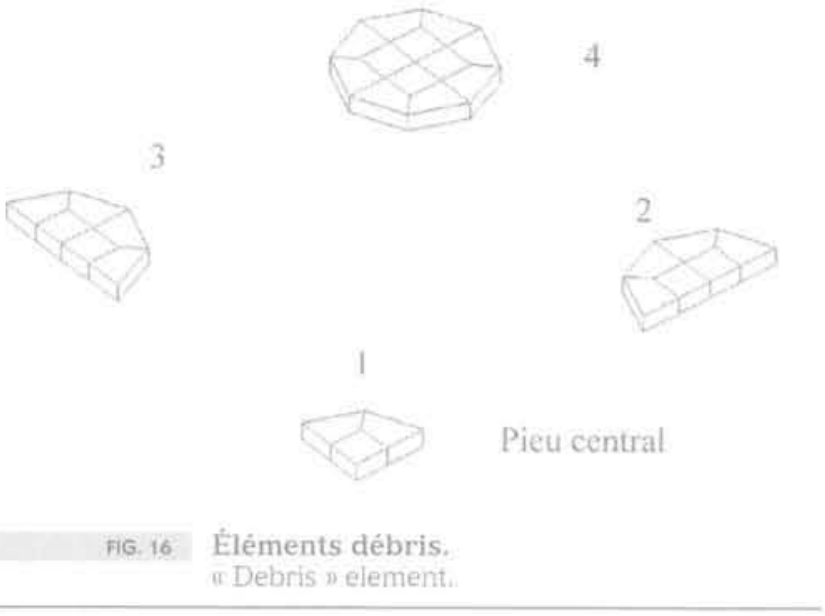

résultats nécessaires afin de comprendre le comportement de ce groupe de pieux, en mettant en évidence dans chaque paragraphe un des paramètres en particulier. Ainsi, nous montrerons comment les differentes distributions de débris sous la pointe des pieux du groupe provoquent des comportements complètement différents, au niveau des tassements. Enfin, nous pourrons comparer les mesures in situ des tassements reels aux premiers pas de chargement.

\section{2,1 \\ Tassements}

Le but de ce travail est d'obtenir des prévisions sur les tassements probables de la pile du pont-canal de Houdeng, afin d'obtenir des indications sur les corrections à apporter pendant la réalisation de l'ouvrage pour rester dans les valeurs de tassements différentiels permis entre chaque pile. Notre simulation nous a permis de comprendre le comportement de ce groupe de pieux et d'estimer les valeurs des tassements, en fonction de différentes distributions des débris sous la pointe de chaque pieu.

A la figure 17 nous avons reporté l'évolution des tassements mesurés à la base de la pile pour les dix simulations. On remarque que l'amplitude des tassements est comprise entre les courbes correspondant à deux cas extrêmes : simulation 1 , débris sous tous les pieux (maximum) et simulation 2, pas de débris (minimum). Les tassements calculés au $6^{\circ}$ pas de chargement sont compris entre 10,2 et $12 \mathrm{~mm}$, et se trouvent encore dans la phase linéaire. On note que la présence ou l'absence de débris sous les pieux périphériques joue un rôle important au niveau des tassements. En effet, dans la simulation 4, nous trouvons des tassements importants. Au contraire, la présence ou l'absence de débris sous le pieu central n'influence pas trop les tassements de la pile. De plus, pour la charge de service, notre systeme se trouve encore en phase linéaire et, après le $7^{\mathrm{c}}$ pas de chargement, apparaissent les premières non-linéarités avec des tassements beaucoup plus importants. Pendant la réalisation du pont. afin de vérifier que les tassements relatifs entre chaque pile ne dépassent pas $10 \mathrm{~mm}$, beaucoup de mesures de tassements seront réalisées après le poussage de chaque tronçon, Nous reportons également les valeurs des tassements calculés avec la méthode pressiométrique pour les groupes de pieux (Frank, 1999), une des plus utilisées et des plus fiables.

Pour la charge de $20000 \mathrm{kN}$, cette méthode donne un tassement à la base de la pile de $4,1 \mathrm{~mm}$, de $9,7 \mathrm{~mm}$ pour la charge de service, de 15,2 pour la charge ultime de $100000 \mathrm{kN}$. Nous reportons au tableau VI les tassements calculés pour l'une des piles avec les deux méthodes. Nous devons remarquer la bonne correspondance entre les deux calculs pour les charges plus 


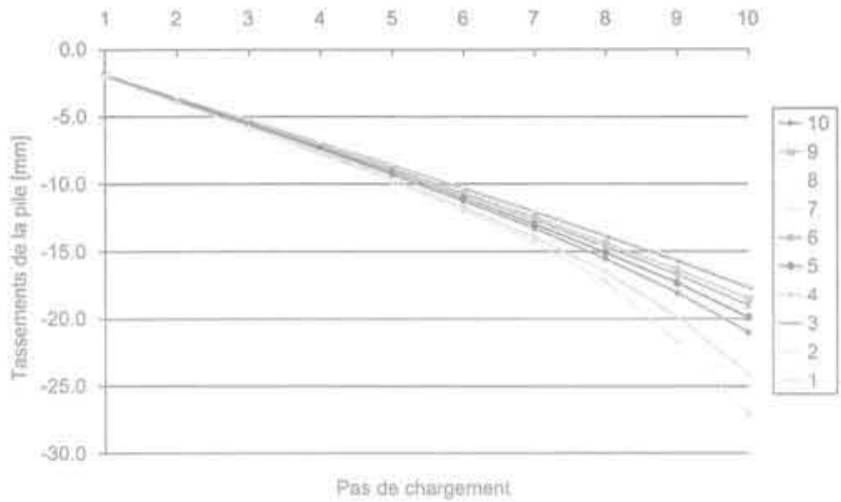

FIG. 17 Tassements à la base de la pile. Plle settlement.

basses, quand on se trouve encore dans un comportement lineaire. Par contre, pour des charges plus importantes, les non-linéarités, accentuées par la présence du débris, sont importantes et influencent beaucoup plus les tassements.

TABLEAUV Tassements calculés avec les différentes méthodes. Settlements estimated with each design method.

\begin{tabular}{c|c|c}
\hline \multirow{2}{*}{ Charge appliquée } & \multicolumn{2}{|c}{ Tassements (mm) } \\
\cline { 2 - 3 } & $\begin{array}{c}\text { Modéle } \\
\text { numerique FEM }\end{array}$ & $\begin{array}{c}\text { Calcul } \\
\text { analytique }\end{array}$ \\
\hline $20000 \mathrm{kN}$ & $3,5-4$ & 4,1 \\
\hline $60000 \mathrm{kN}$ & $10,2-12$ & 9,7 \\
\hline $100000 \mathrm{kN}$ & $17,5-27,1$ & 15,2 \\
\hline
\end{tabular}

\section{achat}

\section{Transfert de la charge par frottement latéral}

Dans le problème étudié, avec les caractéristiques mécaniques des sols et la gẻométrie considérẻe, la charge reprise par frottement latéral dans le groupe de pieux équivaut au moins à $40 \%$ de la charge totale dans presque toutes les simulations, et avec un maximum, pour la simulation 1 , où elle arrive à $66 \%$.

Les pieux périphériques portent la plus grande partie de la charge dans toutes les situations. Cet effet montre que le comportement des groupes de pieux peut être comparé à celui d'un parallélépipède qui se tasse dans son ensemble et qui reprend la charge sur sa surface latérale. Le sol compris entre les pieux tasse avec le radier et les pieux; la résistance par frottement latéral du pieu central est donc peu mobilisée.

A la figure 18 , nous donnons la répartition de la charge totale entre le radier, le frottement latéral et la résistance à la pointe dans la simulation 5 . Le radier reprend une partie de la charge à partir du premier pas ; ensuite sa contribution à la résistance devient constante, démontrant que le groupe de pieux tasse dans son ensemble et que l'influence du sol entre les pieux est minimale.

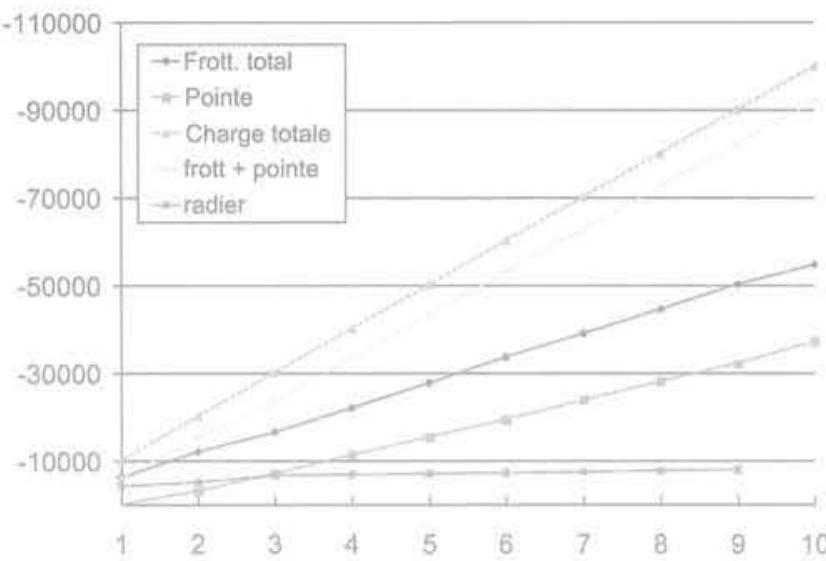

FG. 18 Distribution de la charge [kN] entre les composantes du groupe de pieux.

Redistribution of charges [kN] between each component of pile group.

Un résultat intéressant à mettre en évidence est la façon selon laquelle les contraintes tangentielles verticales se distribuent sur la surface latérale des pieux (Fig. 19, simulation 2: 10" pas). En effet, dans les méthodes analytiques, le calcul de la capacité portante par frottement latéral des pieux suppose une distribution uniforme des contraintes sur la circonférence du pieu. Cette hypothèse est correcte dans le cas d'un pieu isolé sous charge verticale, mais pour un groupe de pieux, la distribution des contraintes tangentielles n'est plus uniforme, surtout pour les pieux extérieurs. Les simulations ont montré des différences importantes de contraintes tangentielles verticales pour les pieux extérieurs du groupe.

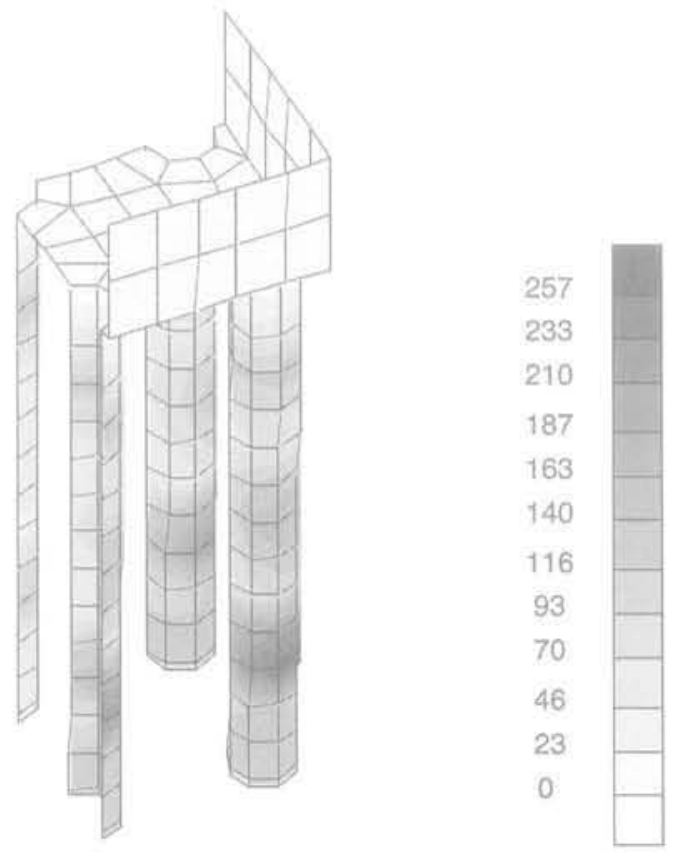

$(\mathrm{kPa})$

FIG. 19 Distribution des contraintes tangentielles verticales sur Ja surface des pieux.

Distribution of vertical tangential stresses alons the pile surface. 
La distribution des $\tau_{y^{\prime}}$ au $6^{e}$ pas de chargement pour la simulation 4, pour les pieux 1,2 et 4 , est donnée à la figure 20 (P2l est la valeur du $\tau$ pour le pieu 2 calculée dans la partie de surface vers l'intérieur du groupe, P2E est la valeur du $\tau$, pour le pieu 2 calculée dans la partie de surface vers l'extérieur du groupe). Le pieu central P1 est caractérisé par une contrainte $\tau$. quasi constante sur la circonférence des pieux. La contrainte $\tau_{\mathrm{y}}$ change sur la hauteur en fonction des différentes couches.
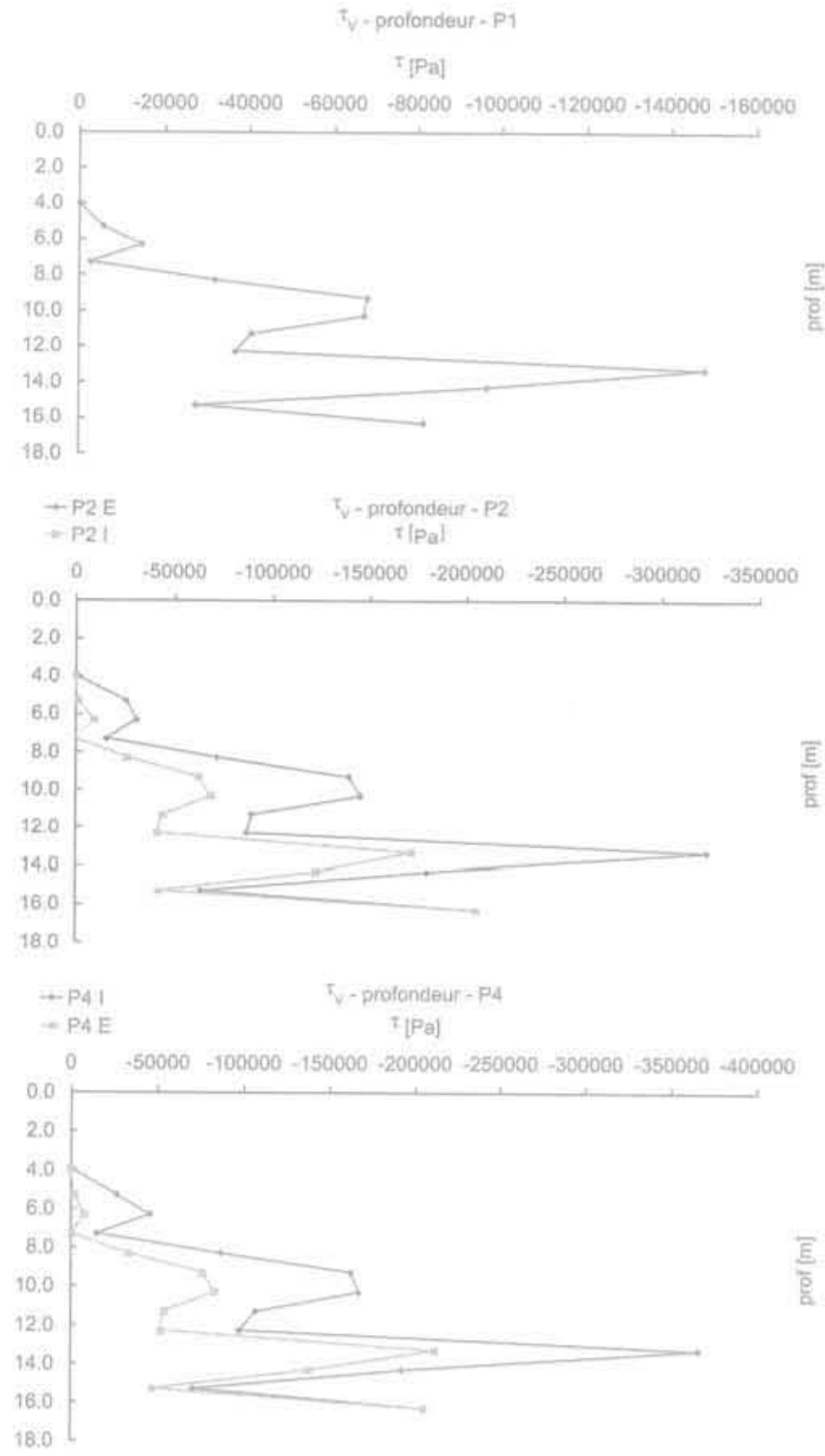

FIG. 20

Contrainte tangentielle verticale mobilisée (SIM 4: 6e pas de chargement). Vertical tangentiel stresses.

L'ordre de grandeur du frottement latéral mobilisé dans le pieu central et dans les pieux latéraux est différent, ainsi qu'entre l'intérieur et l'extérieur de la surface des pieux latéraux. En particulier, on montre que pour la surface intérieure des pieux périphériques et pour le pieu central, des contraintes $\tau_{v}$ du même ordre de grandeur sont mobilisées, alors que sur la surface extérieure des pieux latéraux une contrainte $\tau$, bien plus importante est mobilisée, jusqu'au double de celle à l'intérieur.

\section{6n:az}

\section{Les contraintes dans le béton}

Dans notre travail nous avons remarqué l'importance de l'étude du sol et du contact sol béton. Dans ce paragraphe nous voulons mettre en évidence l'importance d'étudier l'ensemble sol-béton, en particulier afin d'obtenir des indications sur les interactions entre ces deux matériaux. En effet, le code de calcul que nous avons utilisé nous permet de connaitre la distribution des contraintes dans le béton et ainsi d'obtenir des indications sur la structure et son dimensionnement.

La figure 21 donne les contraintes verticales dans le béton pour la simulation 1 au dernier pas de chargement. En utilisant une loi de comportement plus poussée pour le béton, cet outil pourrait être employé pour l'étude des contraintes dans le béton et pour le dimensionnement des structures de fonclation, en permettant aussi des économies importantes de matériaux.
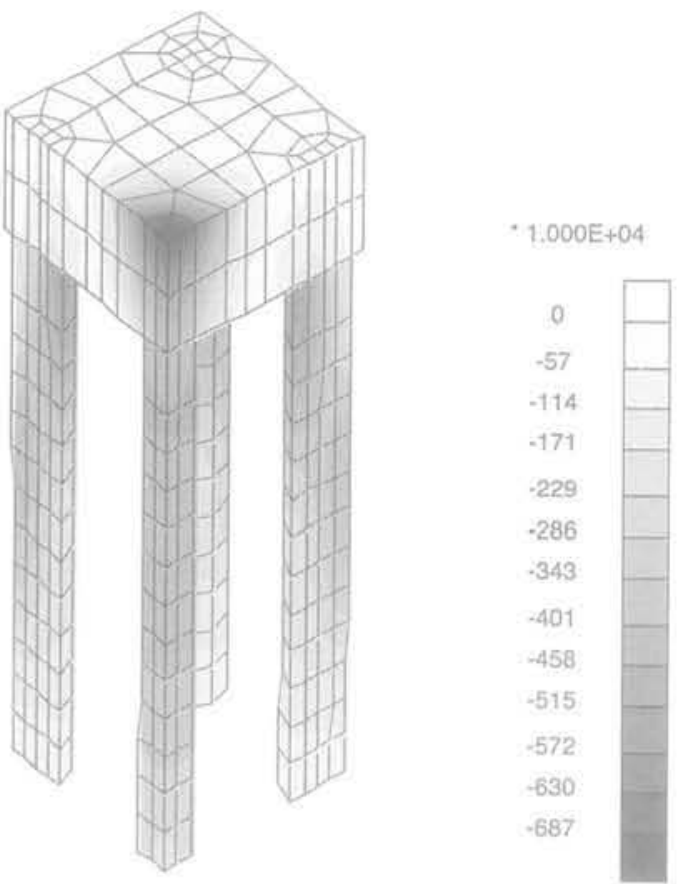

FiG.21 Contraintes verticales dans le béton au dernier pas de chargement (simulation 1). Concrete vertical stresses at the last step of loading (simulation 1):

\section{Conclusion}

Si beaucoup de publications scientifiques concernent le comportement des groupes de pieux, de nombreux aspects restent à ce jour mal compris ou difficiles à prendre en compte dans un dimensionnement.

Notre travail offre une voie possible â suivre : la modélisation numérique, associée à des données expérimentales en nombre suffisant. Par une comparaison permanente entre les mesures in situ réalisées sur les ouvrages et les réponses des modèles numériques, 
nous pourrons obtenir un outil fiable afin de comprendre le fonctionnement réel d'un groupe de pieux. Une simulation tridimensionnelle aux éléments finis nous a permis de comprendre l'ensemble des phénomènes impliqués dans le problème étudié et ainsi de donner une première estimation des tassements prévisibles au pont-canal de Houdeng.

D'un point de vue global, les résultats obtenus ont confirmé le rôle que chaque composante du groupe de pieux (radier, portance à la pointe, frottement latéral) exerce dans la reprise des charges. En particulier, nous avons remarqué que la contribution du radier devient constante après les premiers pas de chargement. La contribution du frottement latéral est ici la première résistance à être mobilisée. Enfin, la contribution de la résistance à la pointe des pieux est mobilisée plus tard, quand les tassements deviennent importants.

Ces simulations ont confirmé l'importance des pieux extérieurs, les plus sollicités pour presque toutes les simulations. De ce fait, il serait intéressant, dans le futur, d'étudier l'influence de la rigidité du radier, en faisant des simulations avec des radiers de différentes épaisseurs et dimensions.

Un autre résultat très intéressant est la différence de distribution des contraintes tangentielles verticales sur la circonférence de chaque pieu. La grande différence que nous avons pu observer entre la partie de la surface à l'intérieur du groupe et celle à l'extérieur (beaucoup plus sollicitée) est une donnée intẻressante afin de mieux comprendre le système étudié. En relation au problème des tassements du pont-canal de Houdeng, nous avons estimé l'influence de la présence des débris sous la pointe des pieux. Les simulations nous ont montré que pour la charge de service de $60000 \mathrm{kN}$ l'influence de cette couche plus faible est bien limitée et les tassements sont compris entre 10,2 et $12 \mathrm{~mm}$. Par contre, pour une charge théorique de $100000 \mathrm{kN}$ l'intervalle est bien plus important (entre 17,5 et $27,1 \mathrm{~mm}$ ), à cause de l'entrée en plasticité pour des charges plus importantes. L'effet des non-linéarités liées aux glissements et aux plastifications est donc très marqué.

Dans la suite, cette étude pourra suivre plusieurs directions : d'une part, l'amélioration du code de calcul en 3D, du générateur de maillage, des aspects purement informatiques, et d'autre part, la réalisation de nouvelles simulations 3D pour différentes situations géotechniques. En particulier, il faudra mettre au point de nouvelles lois de comportement pour les éléments d'interface 3D. La loi de Coulomb offre de bons résultats, mais une loi qui tient compte d'un écrouissage permettrait d'obtenir de meilleurs résultats pour notre travail, comme nous avons pu le constater dans les simulations 2D.

Les directions à suivre sont nombreuses, mais le but est toujours d'arriver à comprendre exactement ce problème qui n'a pas encore été assez ètudié.

\section{Bibliographie}

Barnichon J.D. - a Finite element modelling in structural and petroleum geology x. Thèse de doctorat, Universite de Liège, 1998 .

Boulon M. - «ockélisation numérique 3D du comportement d'un pieu sous effort incliné par la méthode des éléments finis 1 . Intermediaire $n^{\circ} 2$, Rapport Laboratoîre 3S, 1997

Cescotto S., Charlier R. - u Frictional contact finite elements based on mixed variational principles v. Internationa Journal for Numerical methods in Engineering, 36. 1993, p. 1681-1710.

Charlier R. - a Approche unifiée de quelques problèmes non linéaires di mécanique des milieux continus par la méthode des éléments finis n. Thèse de doctorat. Université de Liège. 1987

Charlier R., Habraken A.M.H. - " Numerical modelisation of contact with friction phenomena by the finite element method n. Computer and Geornechanics, vol 9. nos 1 \& 2. 1990
Coduto Donald P. - Foundation Design. principles and practice, and edition. 2001.

Desai C.S. Muqtadir A. - " Three-dimensional analysis of a pile-group foundation $\cdots$. International Journal for Numerical and analytical methods in geomechanics, 10 , 1986 , p. $41-58$

Frank R. - Calcul des fondations superficielles et profondes. Techniques de l'nngénieur, Presses des ponts et chaussées 1999

Frank. R., Zhao S.R. - " Estimation par les paramètres pressiométriques de l'enfoncement sous charge axiale des pieux forés dans les sols fins iv. Bull. liaison labo p. et ch., $n^{5} 119,17-24$; mai-iuin 1982.

Habraken A.M.H., Cescotto S. - " Contact between deformable solids. The fully coupled approach n. Journal of Mathematical and Computer Modelling (Special issue: Recent adivances in contact and impact mechanics), 1986.

L X.L. Bolle A. Charlier R. - a Pont-canal de Houdeng : simulation par éléments finis d'un essai de chargement sur un pieu et du comportement d'un groupe de pieux n. Rapport général final, Université de Liège, novembre 2000.

Ottaviani M. - * Three-dimensional finite etement analysis of vertically loaded pile groups $n$. Géotechnique 25, 2, 1975, p. $159+174$

Poulos H.G., Xu K.J. - « General elastic analysis of piles groups $n$. International Journal for Numerical and analytical methods in geomechanics, 24, 2000, p. 1109-1138.

Poulos H.G. - - Analysis of the settlement of pile groups \%. Geotechnique 18, 4, 1968 .

Van Eekelen H.A.M. $-\alpha$ Isotropic vield surfaces in the three dimensions for use in soil mechanics n. International Journal for Numerical and analytical methods in geomechanics, 4, 1980, p. 98-101.

Viggiani C. - Fondazioni. CUEN, 1994

Zlenkiewicz O.C., Taylor R.L. - The Finite Element Method, 4th edition. 1987. 